\title{
Third-Order Approximate Solution of Chemical Reaction-Diffusion Brusselator System Using Optimal Homotopy Asymptotic Method
}

\author{
Salem Alkhalaf \\ College of Science and Arts, Computer Science Department, Qassim University, Alrass City, Saudi Arabia \\ Correspondence should be addressed to Salem Alkhalaf; s.alkhalaf@qu.edu.sa
}

Received 5 January 2017; Accepted 5 February 2017; Published 22 February 2017

Academic Editor: Boris G. Konopelchenko

Copyright @ 2017 Salem Alkhalaf. This is an open access article distributed under the Creative Commons Attribution License, which permits unrestricted use, distribution, and reproduction in any medium, provided the original work is properly cited.

The objective of this paper is to investigate the effectiveness and performance of optimal homotopy asymptotic method in solving a system of nonlinear partial differential equations. Since mathematical modeling of certain chemical reaction-diffusion experiments leads to Brusselator equations, it is worth demanding a new technique to solve such a system. We construct a new efficient recurrent relation to solve nonlinear Brusselator system of equations. It is observed that the method is easy to implement and quite valuable for handling nonlinear system of partial differential equations and yielding excellent results at minimum computational cost. Analytical solutions of Brusselator system are presented to demonstrate the viability and practical usefulness of the method. The results reveal that the method is explicit, effective, and easy to use.

\section{Introduction}

The Brusselator model, the nonlinear system of partial differential equations, arises in the modeling of certain chemical reaction-diffusion processes. This Brusselator reactiondiffusion model plays a substantial role in the study of cooperative processes of chemical kinetics. This system occurs in a large number of physical problems. It arises in the creation of ozone by atomic oxygen through a triple collision, in enzymatic reactions, and in plasma and laser physics in numerous coupling among models [1]. A pair of variables are involved in dealing with these chemical reactions; intermediates with input and output chemicals, whose concentrations are likely to be controlled during the reaction process, are substantial under quite genuine conditions and are discussed by Nicolis and Prigogine in $[2,3]$. This model has been revealed as the trimolecular model [4].

The two-dimensional nonlinear reaction-diffusion Brusselator system is

$$
\begin{aligned}
& \frac{\partial}{\partial t} u(x, y, t)=u^{2} v-(A+1) u+\mu\left(\frac{\partial^{2} u}{\partial x^{2}}+\frac{\partial^{2} u}{\partial y^{2}}\right)+B \\
& \frac{\partial}{\partial t} v(x, y, t)=-u^{2} v+A u+\mu\left(\frac{\partial^{2} v}{\partial x^{2}}+\frac{\partial^{2} v}{\partial y^{2}}\right)
\end{aligned}
$$

subject to the initial condition:

$$
\begin{aligned}
& u(x, y, 0)=h(x, y), \\
& v(x, y, 0)=g(x, y),
\end{aligned}
$$

where $u(x, y, t), v(x, y, t)$ are unknown functions representing the dimensionless concentrations of two reactants, $x, y$, and $t$ denote spatial and temporal independent variables, respectively, $A$ and $B$ are constant concentrations of the two reactants, $\mu$ represent the diffusion coefficient, and $h$ and $k$ are known functions. It is evident that, for small values of diffusion coefficient $\mu$, steady state solution of Brusselator system converges to the equilibrium point $(B, A / B)$ if $1-A+$ $B^{2}>0$. During the last few years, the researchers have keen interest in the existence of solution of the Brusselator reaction model when $1-A+B^{2} \ngtr 0$ [5-7]. In this paper, we have made a successful attempt to find the solution of such types of Brusselator system.

Numerical methods need large size of computational works and generally the consequence of round-off error causes loss of precision in the results for system of nonlinear partial differential equations. Analytical methods mostly used for solving these equations are very restricted and can 
be used in very special cases. Therefore, an optimal technique is required to resolve such circumstances.

The OHAM was recently introduced by Marinca and Herişanu [8-11]. In series of papers, authors [12-16] have applied this method effectively to validate the solutions of currently important problems. Iqbal et al. [12-15] used OHAM for linear and nonlinear differential equations and time dependent partial differential equations. Similarly, Idrees et al. [16] validated OHAM for nonlinear phenomena of fluid mechanics.

In this presentation, we have extended OHAM formulation for system of partial differential equations. Particularly, the extended formulation is demonstrated by illustrative example of nonlinear Brusselator system partial differential equations.

\section{OHAM Formulation for Brusselator System}

The optimal homotopy asymptotic method (OHAM) is given in [8-16]; we formulate this method for fractional order partial differential equations in the following steps.

(a) Write the governing partial differential equation system as

$\frac{\partial}{\partial t} u(x, y, t)=u^{2} v-(A+1) u+\mu\left(\frac{\partial^{2} u}{\partial x^{2}}+\frac{\partial^{2} u}{\partial y^{2}}\right)+B$

$\frac{\partial}{\partial t} v(x, y, t)=-u^{2} v+A u+\mu\left(\frac{\partial^{2} v}{\partial x^{2}}+\frac{\partial^{2} v}{\partial y^{2}}\right)$,

subject to the initial condition:

$$
\begin{aligned}
& u(x, y, 0)=h(x, y), \\
& v(x, y, 0)=g(x, y),
\end{aligned}
$$

where $u(x, y, t), v(x, y, t)$ are unknown functions; $x, y$, and $t$ denote spatial and temporal independent variables, respectively.

(b) Construct an optimal homotopy for system of partial differential equations, $\phi_{1}(x, y, t ; p): \Omega \times[0,1] \rightarrow$ $R, \phi_{2}(x, y, t ; p): \psi \times[0,1] \rightarrow R$, which satisfies

$$
\begin{aligned}
& H\left(\phi_{1}(x, y, t ; p), p\right)=(1-p) \frac{\partial}{\partial t} \phi_{1}(x, y, t) \\
& -H_{1}(x, y, t ; p)\left(\frac{\partial \phi_{1}}{\partial t}-\phi_{1}^{2} \phi_{2}+(A+1) \phi_{1}\right. \\
& \left.-\mu\left(\frac{\partial^{2} \phi_{1}}{\partial x^{2}}+\frac{\partial^{2} \phi_{1}}{\partial y^{2}}\right)-B\right)=0 \\
& H\left(\phi_{2}(x, y, t ; p), p\right)=(1-p) \frac{\partial}{\partial t} \phi_{2}(x, y, t) \\
& -H_{2}(x, y, t ; p)\left(\frac{\partial \phi_{2}}{\partial t}-\phi_{1}^{2} \phi_{2}-A \phi_{1}\right. \\
& \left.-\mu\left(\frac{\partial^{2} \phi_{1}}{\partial x^{2}}+\frac{\partial^{2} \phi_{1}}{\partial y^{2}}\right)\right)=0
\end{aligned}
$$

where $p \in[0,1]$ is an embedding parameter, $H_{1}(x$, $y, t ; p)$ and $H_{2}(x, y, t ; p)$ are nonzero auxiliary functions for $p \neq 0$ and $H_{1}(x, y, t ; 0)=0, H_{2}(x, y, t ; 0)=$ 0 , and, clearly, we have

$$
\begin{aligned}
H_{1}(x, y, t ; p)= & H_{2}(x, y, t ; p) \\
= & p K_{1}\left(x, y, t ; c_{1}\right)+p^{2} K_{2}\left(x, y, t ; c_{2}\right) \\
& +\cdots+p^{m} K_{m}\left(x, y, t ; c_{m}\right)
\end{aligned}
$$

where $c_{1}, c_{2}, c_{3}, \ldots, c_{m}$ are convergence control parameters. The selection of functions $K_{m}\left(x, y, t ; c_{i}\right)$ might be different type of polynomial and so on. It is very important to choose the functions, since the convergence of the solution very much depends on these functions. The auxiliary function $H(x, y, t ; c)$ provides us with a simple way to adjust and control the convergence. It also increases the accuracy of the results and effectiveness of the method $[17,18]$. The convergence control parameters adjust and control the convergence, which provides optimal accuracy and effectiveness of the method. The presence of $c_{i}, i=1,2, \ldots, m$ ensures the fast convergence.

(c) Expand $\phi_{1}(x, y, t ; p, c)$ and $\phi_{2}(x, y, t ; p, c)$ in Taylor's series about $p$, to get approximate solutions as

$$
\begin{gathered}
\phi_{1}\left(x, y, t ; p, c_{i}\right)=u_{0}(r, t)+\sum_{k=1}^{m} u_{k}\left(r, t ; c_{i}\right) p^{k} \\
i=1,2,3, \ldots, m, \\
\phi_{2}\left(x, y, t ; p, c_{i}\right)=v_{0}(r, t)+\sum_{k=1}^{m} v_{k}\left(r, t ; c_{i}\right) p^{k} \\
i=1,2,3, \ldots, m .
\end{gathered}
$$

(i) It has been observed that the convergence of the series (8) and (9) depends upon the convergence control parameters.

(ii) If it converges at $p=1$, one has

$$
\begin{array}{ll}
\widetilde{u}\left(r, t ; c_{i}\right)=u_{0}(r, t)+\sum_{k=1}^{m} u_{k}\left(r, t ; c_{i}\right) & \\
& i=1,2,3, \ldots, m, \\
\widetilde{v}\left(r, t ; c_{i}\right)=v_{0}(r, t)+\sum_{k=1}^{m} v_{k}\left(r, t ; c_{i}\right) & \\
i=1,2,3, \ldots, m .
\end{array}
$$


(d) Equate the coefficients of like powers of $p$ after substituting (8) and (9) in (5) and (6), respectively; one can get zeroth-order system given by (12), firstorder and second-order system given by (13)-(14), respectively, and the higher order system if needed:

$$
\begin{aligned}
& p^{0}: \quad \frac{\partial}{\partial t} u_{0}(x, y, t)=0 \\
& \frac{\partial}{\partial t} v_{0}(x, y, t)=0 \\
& +\mu c_{1} \frac{\partial^{2} u_{0}}{\partial x^{2}}-\left(1+c_{1}\right) \frac{\partial u_{0}}{\partial t}+\frac{\partial u_{1}}{\partial t}=0 \\
& A c_{1} u_{0}-c_{1} u_{0}^{2} v_{0}+\mu c_{1} \frac{\partial^{2} v_{0}}{\partial y^{2}}+\mu c_{1} \frac{\partial^{2} v_{0}}{\partial x^{2}} \\
& -\left(1+c_{1}\right) \frac{\partial v_{0}}{\partial t}+\frac{\partial v_{1}}{\partial t}=0
\end{aligned}
$$

$$
\begin{aligned}
p^{2}: \quad B c_{2} & -c_{2} u_{0}-A c_{2} u_{0}-c_{1} u_{1}-A c_{1} u_{1}+c_{2} u_{0}^{2} v_{0} \\
& +2 c_{1} u_{0} u_{1} v_{0}+c_{1} u_{0}^{2} v_{1}+\mu c_{2} \frac{\partial^{2} u_{0}}{\partial y^{2}}+\mu c_{1} \frac{\partial^{2} u_{1}}{\partial y^{2}} \\
& +\mu c_{2} \frac{\partial^{2} u_{0}}{\partial x^{2}}+\mu c_{1} \frac{\partial^{2} u_{1}}{\partial x^{2}}-c_{2} \frac{\partial u_{0}}{\partial t}-\left(1+c_{1}\right) \frac{\partial u_{1}}{\partial t} \\
& +\frac{\partial u_{2}}{\partial t}=0
\end{aligned}
$$

$$
\begin{gathered}
A c_{2} u_{0}+A c_{1} u_{1}-c_{2} u_{0}^{2} v_{0}-2 c_{1} u_{0} u_{1} v_{0}-c_{1} u_{0}^{2} v_{1} \\
+\frac{1}{4} c_{2} \frac{\partial^{2} v_{0}}{\partial y^{2}}+\mu c_{1} \frac{\partial^{2} v_{1}}{\partial y^{2}}+\mu c_{2} \frac{\partial^{2} v_{0}}{\partial x^{2}}+\mu c_{1} \frac{\partial^{2} v_{1}}{\partial x^{2}} \\
-c_{2} \frac{\partial v_{0}}{\partial t}-\left(1+c_{1}\right) \frac{\partial v_{1}}{\partial t}+\frac{\partial v_{2}}{\partial t}=0
\end{gathered}
$$

and so on.

(e) The above system of nonlinear equations, that is, zeroth-order, first-order, and higher order systems (if needed), can be easily solved. Put these solutions of different order problems in (10) and (11); one can obtain the approximate solutions $\widetilde{u}\left(r, t ; c_{i}\right)$ and $\widetilde{v}\left(r, t ; c_{i}\right)$, respectively.

(f) Determine the convergence control parameters, $c_{1}, c_{2}, c_{3}, \ldots$, by using one of the methods given in [8-11]. Using auxiliary constants in (10) and (11), one can get the approximate solutions $u(x, y, t)$ and $v(x, y, t)$, respectively.

\section{Application}

Example 1. Let us consider with $A=1, \mu=1 / 4, B=0$, $h(x, y)=e^{(-x-y)}$, and $g(x, y)=e^{(x+y)}$ two-dimensional Brusselator system (1) can be written in the following form:

$$
\begin{aligned}
& \frac{\partial u(x, y, t)}{\partial t}=u^{2} v-2 u+\frac{1}{4}\left(\frac{\partial^{2} u}{\partial x^{2}}+\frac{\partial^{2} u}{\partial y^{2}}\right), \\
& \frac{\partial v(x, y, t)}{\partial t}=u-u^{2} v+\frac{1}{4}\left(\frac{\partial^{2} v}{\partial x^{2}}+\frac{\partial^{2} v}{\partial y^{2}}\right),
\end{aligned}
$$

subject to the initial conditions:

$$
\begin{aligned}
u(x, y, 0) & =e^{(-x-y)}, \\
v(x, y, 0) & =e^{(x+y)}, \\
(x, y, t) & \in \mathbb{R}^{2} \times[0,2] .
\end{aligned}
$$

The exact solutions of (15) is found to be [19]

$$
\begin{aligned}
& u(x, y, t)=e^{(-x-y-t / 2)}, \\
& v(x, y, t)=e^{(x+y+t / 2)} .
\end{aligned}
$$

OHAM formulation presented in Section 2 generates the series of problems, which can be written as

$$
\begin{aligned}
& \frac{\partial}{\partial t} u_{0}(x, y, t)=0 \\
& \frac{\partial}{\partial t} v_{0}(x, y, t)=0 \\
& -2 c_{1} u_{0}+c_{1} u_{0}^{2} v_{0}+\frac{1}{4} c_{1} \frac{\partial^{2} u_{0}}{\partial y^{2}}+\frac{1}{4} c_{1} \frac{\partial^{2} u_{0}}{\partial x^{2}} \\
& -\left(1+c_{1}\right) \frac{\partial u_{0}}{\partial t}+\frac{\partial u_{1}}{\partial t}=0 \\
& -c_{1} u_{0}-c_{1} u_{0}^{2} v_{0}+\frac{1}{4} c_{1} \frac{\partial^{2} v_{0}}{\partial y^{2}}+\frac{1}{4} c_{1} \frac{\partial^{2} v_{0}}{\partial x^{2}}-\left(1+c_{1}\right) \frac{\partial v_{0}}{\partial t} \\
& +\frac{\partial v_{1}}{\partial t}=0 \\
& -2 c_{2} u_{0}-2 c_{1} u_{1}+c_{2} u_{0}^{2} v_{0}+2 c_{1} u_{0} u_{1} v_{0}+c_{1} u_{0}^{2} v_{1} \\
& +\frac{1}{4} c_{2} \frac{\partial^{2} u_{0}}{\partial y^{2}}+\frac{1}{4} c_{1} \frac{\partial^{2} u_{1}}{\partial x^{2}}-c_{2} \frac{\partial u_{0}}{\partial t}-\left(1+c_{1}\right) \frac{\partial u_{1}}{\partial t} \\
& +\frac{\partial u_{2}}{\partial t}=0 \\
& -c_{2} u_{0}+c_{1} u_{1}-c_{2} u_{0}^{2} v_{0}-2 c_{1} u_{0} u_{1} v_{0}-c_{1} u_{0}^{2} v_{1} \\
& +\frac{1}{4} c_{2} \frac{\partial^{2} v_{0}}{\partial y^{2}}+\frac{1}{4} c_{1} \frac{\partial^{2} v_{1}}{\partial y^{2}}+\frac{1}{4} c_{2} \frac{\partial^{2} v_{0}}{\partial x^{2}}+\frac{1}{4} c_{1} \frac{\partial^{2} v_{1}}{\partial x^{2}} \\
& -c_{2} \frac{\partial v_{0}}{\partial t}-\left(1+c_{1}\right) \frac{\partial v_{1}}{\partial t}+\frac{\partial v_{2}}{\partial t}=0
\end{aligned}
$$




$$
\begin{aligned}
- & 2 c_{3} u_{0}-2 c_{2} u_{1}-2 c_{1} u_{2}+c_{3} u_{0}^{2} v_{0}+2 c_{2} u_{0} u_{1} v_{0}+c_{1} u_{1}^{2} v_{0} \\
+ & 2 c_{1} u_{0} u_{2} v_{0}+c_{2} u_{0}^{2} v_{1}+2 c_{1} u_{0} u_{1} v_{1}+c_{1} u_{0}^{2} v_{2} \\
+ & \frac{1}{4} c_{3} \frac{\partial^{2} u_{0}}{\partial y^{2}}+\frac{1}{4} c_{2} \frac{\partial^{2} u_{1}}{\partial y^{2}}+\frac{1}{4} c_{1} \frac{\partial^{2} u_{2}}{\partial y^{2}}+\frac{1}{4} c_{3} \frac{\partial^{2} u_{0}}{\partial x^{2}} \\
+ & \frac{1}{4} c_{2} \frac{\partial^{2} u_{1}}{\partial x^{2}}+\frac{1}{4} c_{1} \frac{\partial^{2} u_{2}}{\partial x^{2}}+c_{3} \frac{\partial u_{0}}{\partial t}-c_{2} \frac{\partial u_{1}}{\partial t} \\
& -\left(1+c_{1}\right) \frac{\partial u_{2}}{\partial t}+\frac{\partial u_{3}}{\partial t}=0, \\
c_{3} u_{0} & +c_{2} u_{1}+c_{1} u_{2}-c_{3} u_{0}^{2} v_{0}-2 c_{2} u_{0} u_{1} v_{0}-c_{1} u_{1}^{2} v_{0} \\
& -2 c_{1} u_{0} u_{2} v_{0}-c_{2} u_{0}^{2} v_{1}-2 c_{1} u_{0} u_{1} v_{1}-c_{1} u_{0}^{2} v_{2} \\
+ & \frac{1}{4} c_{3} \frac{\partial^{2} v_{0}}{\partial y^{2}}+\frac{1}{4} c_{2} \frac{\partial^{2} v_{1}}{\partial y^{2}}+\frac{1}{4} c_{1} \frac{\partial^{2} v_{2}}{\partial y^{2}}+\frac{1}{4} c_{3} \frac{\partial^{2} v_{0}}{\partial x^{2}} \\
+ & \frac{1}{4} c_{2} \frac{\partial^{2} v_{1}}{\partial x^{2}}+\frac{1}{4} c_{1} \frac{\partial^{2} v_{2}}{\partial x^{2}}-c_{3} \frac{\partial v_{0}}{\partial t}-c_{2} \frac{\partial v_{1}}{\partial t} \\
+ & \left(1+c_{1}\right) \frac{\partial v_{2}}{\partial t}+\frac{\partial v_{3}}{\partial t}=0 .
\end{aligned}
$$

The above zeroth-order, first-order, second-order, and third-order problems are given in (18) which can easily be solved. We get

$$
\begin{aligned}
u_{0}(x, y, t) & =e^{(-x-y)}, \\
v_{0}(x, y, t) & =e^{(x+y)}, \\
u_{1}(x, y, t) & =\frac{t}{2} e^{(-x-y)} c_{1}, \\
v_{1}(x, y, t) & =\frac{-t}{2} e^{(x+y)} c_{1}, \\
u_{2}(x, y, t) & =\frac{t}{8} e^{(-x-y)}\left(4 c_{1}+4 c_{1}^{2}+t c_{1}^{2}+4 c_{2}\right), \\
v_{2}(x, y, t) & =\frac{t}{8} e^{(x+y)}\left(-4 c_{1}-4 c_{1}^{2}+t c_{1}^{2}-4 c_{2}\right), \\
u_{3}(x, y, t) & =\frac{t}{48} e^{(-x-y)}\left(24 c_{1}+48 c_{1}^{2}+12 t c_{1}^{2}+24 c_{1}^{3}\right. \\
+12 t c_{1}^{3} & \left.+t^{2} c_{1}^{3}+24 c_{2}+48 c_{1} c_{2}+12 t c_{1} c_{2}+24 c_{3}\right), \\
v_{3}(x, y, t) & =\frac{-t}{48} e^{(x+y)}\left(24 c_{1}+48 c_{1}^{2}-12 t c_{1}^{2}+24 c_{1}^{3}\right. \\
-12 t c_{1}^{3} & \left.+t^{2} c_{1}^{3}+24 c_{2}+48 c_{1} c_{2}-12 t c_{1} c_{2}+24 c_{3}\right)
\end{aligned}
$$

Substituting (19) in (10) and (11), respectively, we obtain

$$
\begin{aligned}
& u(x, y, t)=\frac{e^{-x-y}}{48}\left(18 t(4+t) c_{1}^{2}\right. \\
& \quad+t c_{1}^{3}\left(24+12 t+t^{2}\right)+12 t c_{1}\left(6+(4+t) c_{2}\right)
\end{aligned}
$$

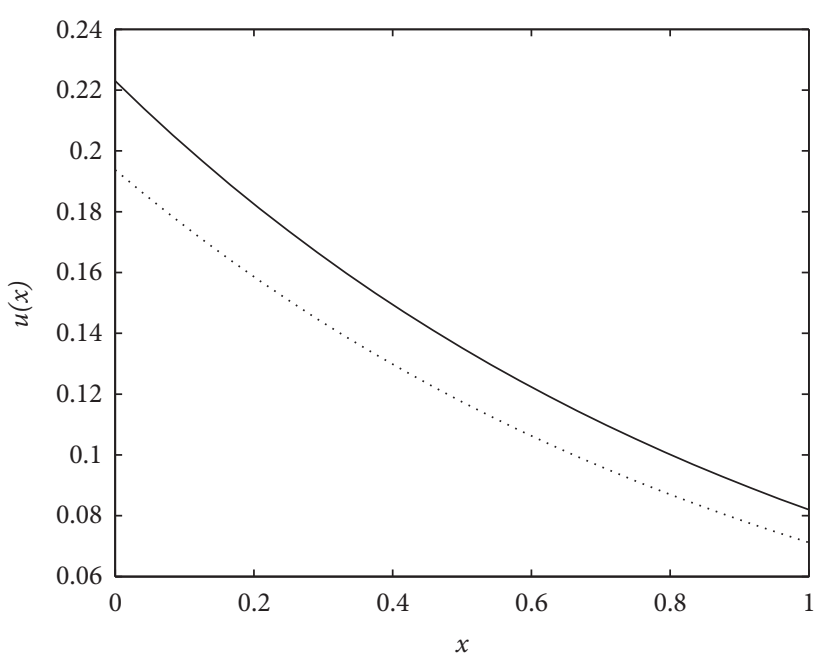

..... First-order solution

— Exact solution

FIGURE 1: Convergence of first-order solution of $u(x, y, t)$ at $t=y=$ 1.

$$
\begin{aligned}
& \left.+24\left(2+2 t c_{2}+t c_{3}\right)\right), \\
& v(x, y, t)=\frac{-e^{x y}}{48}\left(-18 t(-4+t) c_{1}^{2}\right. \\
& \quad+t c_{1}^{3}\left(24-12 t+t^{2}\right)-12 t c_{1}\left(-6+(-4+t) c_{2}\right) \\
& \left.\quad+24\left(-2+2 t c_{2}+t c_{3}\right)\right) .
\end{aligned}
$$

Auxiliary constants shown in (20) can be found by using step (f) in Section 2.

\section{Results and Discussions}

The formulation of OHAM for two-dimensional nonlinear Brusselator system with $A=1, B=0$, and $\mu=0.25$ is presented in Section 2 and the demonstration of the formulation is presented in Example 1.

The third-order approximate solutions of $u(x, y, t)$, $v(x, y, t)$ are given in (19). These solutions depend upon the optimal convergence control parameters which are given in Table 1 . The simplicity and accuracy of the presented method are illustrated by computing $\left|u_{\text {Exact }}-u_{\text {OHAM }}\right|,\left|v_{\text {Exact }}-v_{\text {OHAM }}\right|$. Tables 2 and 3 at different gird points show the comparisons of absolute error of $u(x, y, t)$ and $v(x, y, t)$ between OHAM and the exact solutions, respectively. In this study, only up to third-order solutions are considered. From Tables 2 and 3 , it is clear that OHAM achieves accurate solutions at only third-order term of approximation without any spatial discretization. Thus the third-order approximate solutions of Brusselator reaction-diffusion system converge.

Figures 1, 2, and 3 show the convergence of firstorder, second-order, and third-order approximate solutions of $u(x, y, t)$ obtained by OHAM, respectively. Figures 4,5 , 


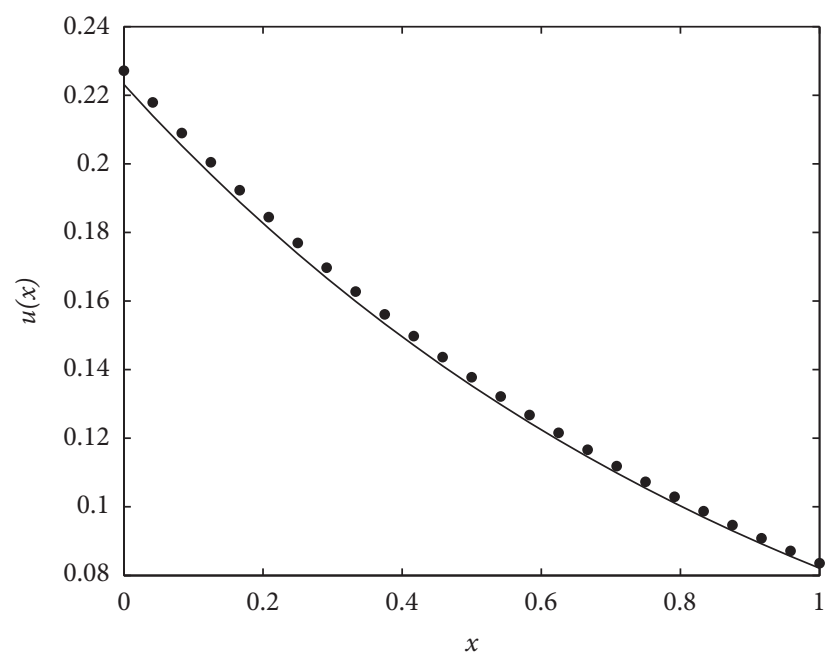

- Second-order solution

Exact solution

Figure 2: Convergence of second-order solution of $u(x, y, t)$ at $t=$ $y=1$.

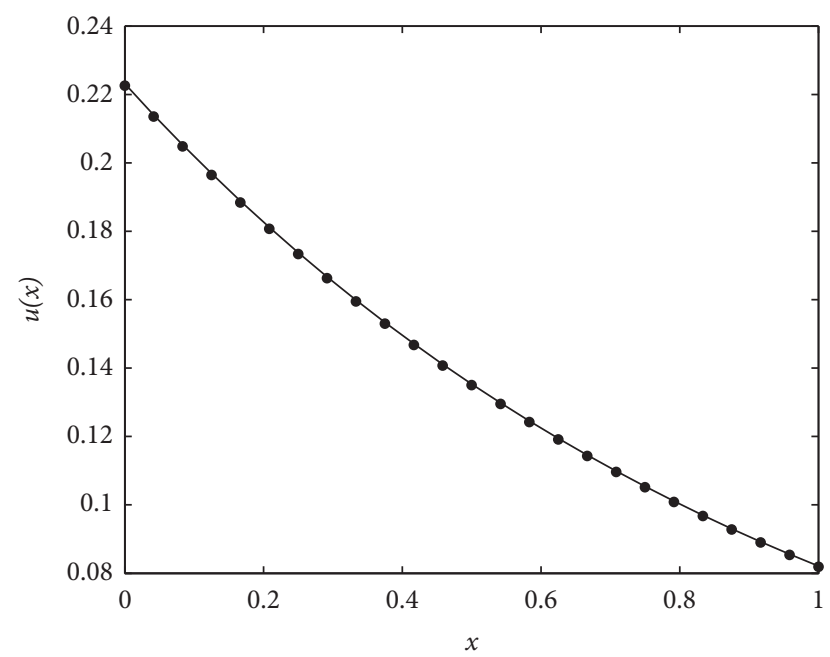

- Third-order solution

- Exact solution

Figure 3: Convergence of third-order solution of $u(x, y, t)$ at $t=$ $y=1$.

TABLE 1: Convergence control parameters of example for $u(x, y, t)$ and $v(x, y, t)$.

\begin{tabular}{cccc}
\hline & $c_{1}$ & $c_{2}$ & $c_{3}$ \\
\hline$u(x, y, t)$ & -0.945983141 & 0.0083062488 & 0.0004400396 \\
$v(x, y, t)$ & -1.0838346562 & 0.0032355216 & -0.0012528760 \\
\hline
\end{tabular}

and 6 show the convergence of first-order, second-order, and third-order approximate solutions of $v(x, y, t)$ obtained by

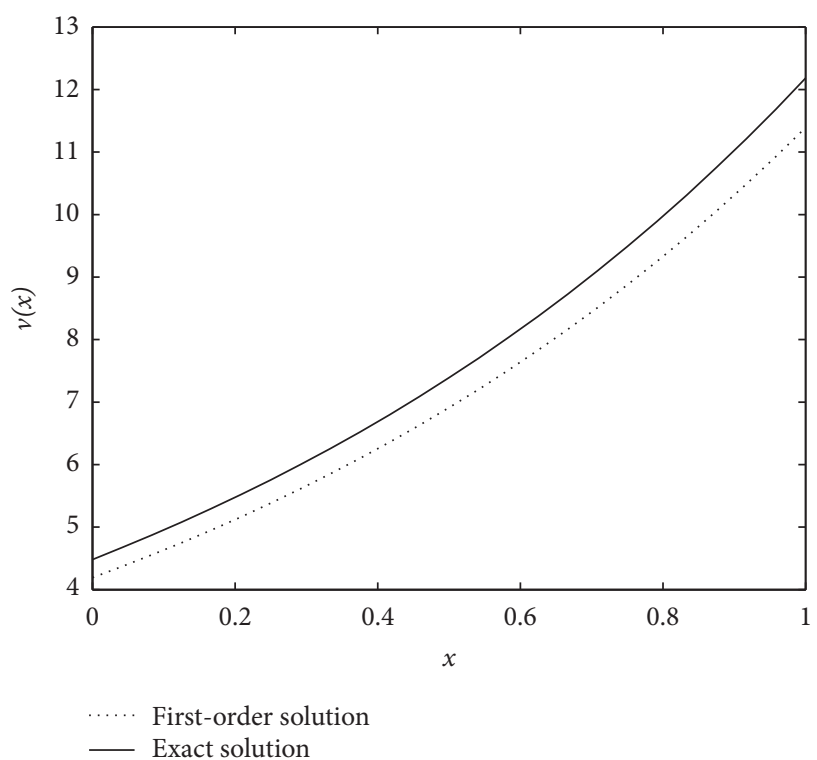

FIGURE 4: Convergence of first-order solution of $v(x, y, t)$ at $t=y=$

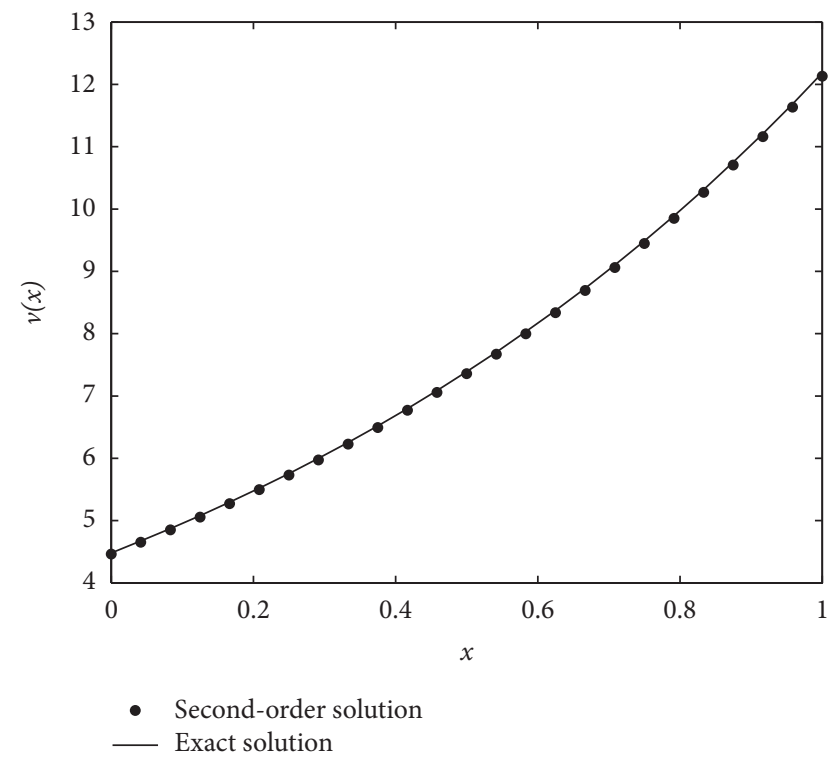

FIgURE 5: Convergence of second-order solution of $v(x, y, t)$ at $t=$ $y=1$.

OHAM, respectively. Figure 7 shows the error convergence with the order of approximation of $u(x, y, t)$ and $v(x, y, t)$. It is clear from the figures that the behaviour of approximate solutions is highly same as exact solution. It can also be clear from Figures 3 and 6 that third-order approximate solution converges and there is no need to compute extra terms when OHAM is used. It is observed that as we move along the domain we get consistent accuracy. It can also be observed that approximate solutions by formulation are in excellent agreement with the exact solutions. Thus the series 
TABLE 2: Comparison of absolute errors of $u(x, y, t)$ at different orders of approximation.

\begin{tabular}{|c|c|c|c|c|c|c|c|}
\hline \multirow{2}{*}{$t$} & \multirow{2}{*}{$x$} & \multirow{2}{*}{$y$} & \multirow{2}{*}{ OHAM } & \multirow{2}{*}{ Exact } & \multicolumn{3}{|c|}{ Absolute error $=\left|u_{\text {exact }}-u_{\text {OHAM }}\right|$} \\
\hline & & & & & 1st-order error & 2nd-order error & 3rd-order error \\
\hline \multirow[t]{3}{*}{0.25} & 0.25 & 0.25 & 0.535285 & 0.535261 & 0.000451741 & 0.000544293 & $2.31736 \times 10^{-5}$ \\
\hline & & 0.5 & 0.41688 & 0.416862 & 0.000351816 & 0.000423896 & $1.80476 \times 10^{-5}$ \\
\hline & & 0.75 & 0.324667 & 0.324652 & 0.000273995 & 0.000330131 & $1.40555 \times 10^{-5}$ \\
\hline \multirow[t]{3}{*}{0.5} & 0.5 & 0.25 & 0.367815 & 0.367879 & 0.00722559 & 0.000930733 & $6.44165 \times 10^{-5}$ \\
\hline & & 0.5 & 0.286455 & 0.286505 & 0.00562729 & 0.000724856 & $5.01676 \times 10^{-5}$ \\
\hline & & 0.75 & 0.223091 & 0.22313 & 0.00438254 & 0.000564518 & $3.90706 \times 10^{-5}$ \\
\hline \multirow[t]{3}{*}{0.75} & 0.75 & 0.25 & 0.367815 & 0.367879 & 0.00722559 & 0.000930733 & $6.44165 \times 10^{-5}$ \\
\hline & & 0.5 & 0.286455 & 0.286505 & 0.00562729 & 0.000724856 & $5.01676 \times 10^{-5}$ \\
\hline & & 0.75 & 0.223091 & 0.22313 & 0.00438254 & 0.000564518 & $3.90706 \times 10^{-5}$ \\
\hline
\end{tabular}

TABLE 3: Comparison of absolute errors of $v(x, y, t)$ at different orders of approximation.

\begin{tabular}{|c|c|c|c|c|c|c|c|}
\hline \multirow{2}{*}{$t$} & \multirow{2}{*}{$x$} & \multirow{2}{*}{$y$} & \multirow{2}{*}{ OHAM } & \multirow{2}{*}{ Exact } & \multicolumn{3}{|c|}{ Absolute error $=\left|v_{\text {exact }}-v_{\text {OHAM }}\right|$} \\
\hline & & & & & 1st-order error & 2nd-order error & 3rd-order error \\
\hline \multirow[t]{3}{*}{0.01} & 0.2 & 0.25 & 1.57619 & 1.57617 & 0.000637758 & 0.0000770919 & $1.81338 \times 10^{-5}$ \\
\hline & & 0.5 & 2.02387 & 2.02385 & 0.000818897 & 0.000098988 & $2.32843 \times 10^{-5}$ \\
\hline & & 0.75 & 2.5987 & 2.59867 & 0.00105149 & 0.000127103 & $2.98976 \times 10^{-5}$ \\
\hline \multirow[t]{3}{*}{0.02} & 0.4 & 0.25 & 1.93484 & 1.93479 & 0.00150979 & 0.000180195 & $4.29601 \times 10^{-5}$ \\
\hline & & 0.5 & 2.48438 & 2.48432 & 0.00193861 & 0.000231375 & $5.51618 \times 10^{-5}$ \\
\hline & & 0.75 & 3.19 & 3.18993 & 0.00248922 & 0.000297091 & $7.08292 \times 10^{-5}$ \\
\hline \multirow[t]{3}{*}{0.03} & 0.8 & 0.25 & 2.90093 & 2.90084 & 0.00327045 & 0.000385405 & $9.32357 \times 10^{-5}$ \\
\hline & & 0.5 & 3.72487 & 3.72475 & 0.00419935 & 0.00049487 & $1.19717 \times 10^{-4}$ \\
\hline & & 0.75 & 4.78283 & 4.78267 & 0.00539207 & 0.000635425 & $1.5372 \times 10^{-4}$ \\
\hline
\end{tabular}

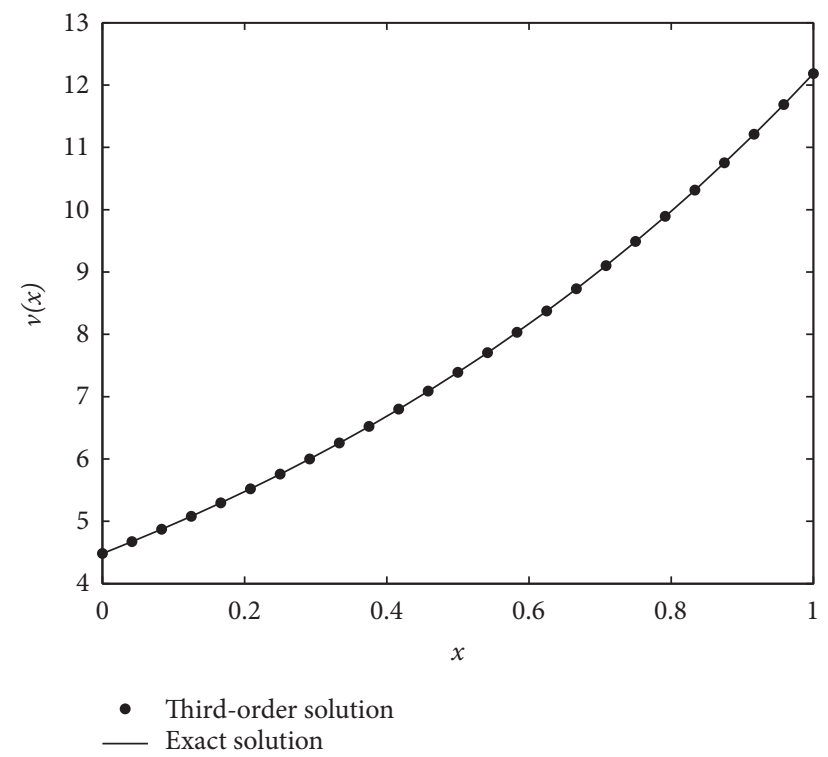

FIGURE 6: Convergence of third-order solution of $v(x, y, t)$ at $t=$ $y=1$.

solutions for fractional equations converge. Results indicate the performance of the method nonlinear system of partial differential equations in precisely approximating the solution at less computational cost.

\section{Conclusion}

In this work, we employed a new powerful semianalytic technique, optimal homotopy asymptotic method to solve Brusselator reaction-diffusion system. The objective of this work is to illustrate the usefulness of the technique. This technique is simple in applicability, as it does not need discretization like numerical methods. Furthermore, this technique delivers an appropriate way to control the convergence by optimally shaping the convergence control parameters. Additionally, this method converges rapidly at lower order of approximations. Therefore, OHAM exhibits its concealed supremacy and is latent for the solution of nonlinear problems in real life applications. One substantial objective of this effort is the investigation of convergence and practicality of the method. By using this method, we acquire a new effective recurrent relation to solve nonlinear Brusselator system. The results demonstrate that the method is a prevailing mathematical tool for solving systems of nonlinear partial differential equations having extensive applications in science and engineering. 

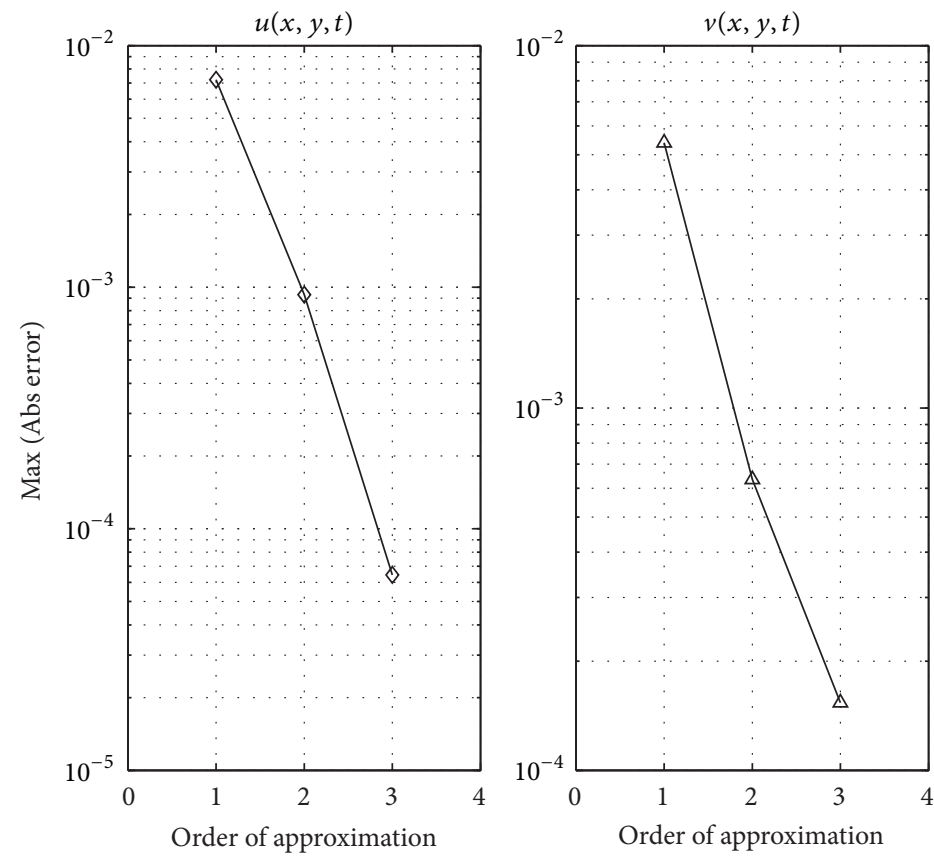

FIGURE 7: Error convergence with the order of approximation of $u(x, y, t)$ and $v(x, y, t)$.

\section{Competing Interests}

The author declares that there is no conflict of interests regarding the publication of this paper.

\section{References}

[1] S. Kumar, Y. Khan, and A. Yildirim, "A mathematical modeling arising in the chemical systems and its approximate numerical solution," Asia-Pacific Journal of Chemical Engineering, vol. 7, no. 6 , pp. 835-840, 2012.

[2] G. Nicolis and I. Prigogine, Self-Organization in Nonequilibrium Systems, John Wiley \& Sons, New York, NY, USA, 1977.

[3] I. Prigogine and R. Lefever, "Symmetry breaking instabilities in dissipative systems. II," The Journal of Chemical Physics, vol. 48, no. 4, pp. 1695-1700, 1968.

[4] J. J. Tyson, "Some further studies of nonlinear oscillations in chemical systems," The Journal of Chemical Physics, vol. 58, no. 9, pp. 3919-3930, 1973.

[5] S. Islam, A. Ali, and S. Haq, "A computational modeling of the behavior of the two-dimensional reaction-diffusion Brusselator system," Applied Mathematical Modelling, vol. 34, no. 12, pp. 3896-3909, 2010.

[6] R. C. Mittal and R. Jiwari, "Numerical solution of two-dimensional reaction-diffusion Brusselator system," Applied Mathematics and Computation, vol. 217, no. 12, pp. 5404-5415, 2011.

[7] M. S. H. Chowdhury, T. H. Hassan, and S. Mawa, "A new application of homotopy perturbation method to the reactiondiffusion brusselator model," Procedia-Social and Behavioral Sciences, vol. 8, pp. 648-653, 2010.

[8] V. Marinca and N. Herișanu, "Application of optimal homotopy asymptotic method for solving nonlinear equations arising in heat transfer," International Communications in Heat and Mass Transfer, vol. 35, no. 6, pp. 710-715, 2008.
[9] V. Marinca and N. Herişanu, "Determination of periodic solutions for the motion of a particle on a rotating parabola by means of the optimal homotopy asymptotic method," Journal of Sound and Vibration, vol. 329, no. 9, pp. 1450-1459, 2010.

[10] N. Herişanu and V. Marinca, "Accurate analytical solutions to oscillators with discontinuities and fractional-power restoring force by means of the optimal homotopy asymptotic method," Computers \& Mathematics with Applications, vol. 60, no. 6, pp. 1607-1615, 2010.

[11] V. Marinca and N. Herisanu, "The optimal homotopy asymptotic method for solving Blasius equation," Applied Mathematics and Computation, vol. 231, pp. 134-139, 2014.

[12] S. Iqbal, M. Idrees, A. M. Siddiqui, and A. R. Ansari, "Some solutions of the linear and nonlinear Klein-Gordon equations using the optimal homotopy asymptotic method," Applied Mathematics and Computation, vol. 216, no. 10, pp. 2898-2909, 2010.

[13] S. Iqbal and A. Javed, "Application of optimal homotopy asymptotic method for the analytic solution of singular Lane-Emden type equation," Applied Mathematics and Computation, vol. 217, no. 19, pp. 7753-7761, 2011.

[14] S. Iqbal, A. R. Ansari, A. M. Siddiqui, and A. Javed, "Use of optimal homotopy asymptotic method and Galerkin's finite element formulation in the study of heat transfer flow of a third grade fluid between parallel plates," Journal of Heat Transfer, vol. 133, no. 9, Article ID 091702, 2011.

[15] M. S. Hashmi, N. Khan, and S. Iqbal, "Optimal homotopy asymptotic method for solving nonlinear Fredholm integral equations of second kind," Applied Mathematics and Computation, vol. 218, no. 22, pp. 10982-10989, 2012.

[16] M. Idrees, S. Islam, S. Haq, and S. Islam, "Application of the optimal homotopy asymptotic method to squeezing flow," Computers \& Mathematics with Applications, vol. 59, no. 12, pp. 3858-3866, 2010. 
[17] S. Sarwar, S. Alkhalaf, S. Iqbal, and M. A. Zahid, "A note on optimal homotopy asymptotic method for the solutions of fractional order heat- and wave-like partial differential equations," Computers \& Mathematics with Applications, vol. 70, no. 5, pp. 942-953, 2015.

[18] N. Herisanu, V. Marinca, and G. Madescu, "An analytical approach to non-linear dynamical model of a permanent magnet synchronous generator," Wind Energy, vol. 18, no. 9, pp. 16571670, 2015.

[19] W.-T. Ang, "The two-dimensional reaction-diffusion Brusselator system: a dual-reciprocity boundary element solution," Engineering Analysis with Boundary Elements, vol. 27, no. 9, pp. 897-903, 2003. 


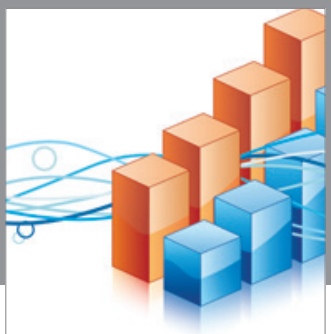

Advances in

Operations Research

vatem alat4

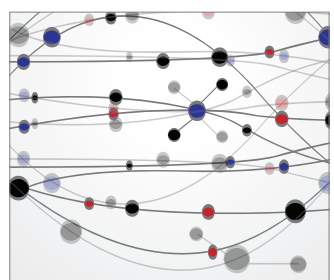

\section{The Scientific} World Journal
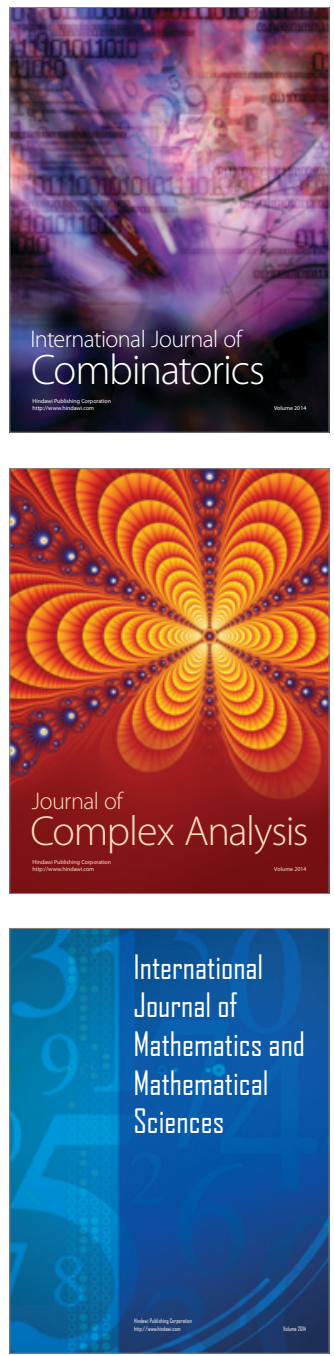
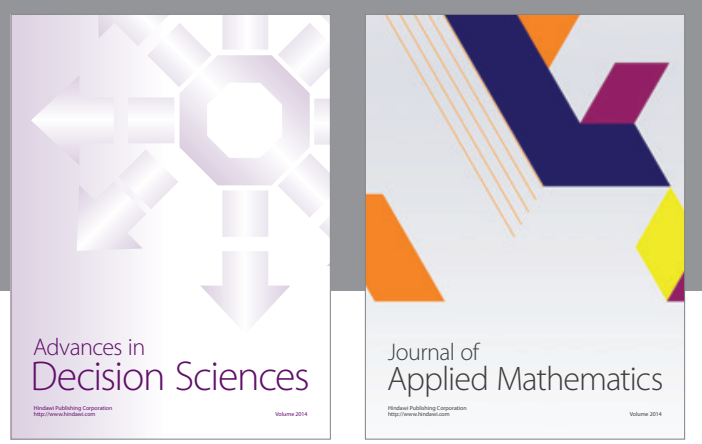

Algebra

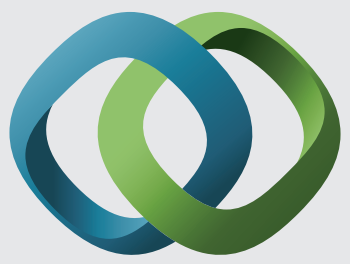

\section{Hindawi}

Submit your manuscripts at

https://www.hindawi.com
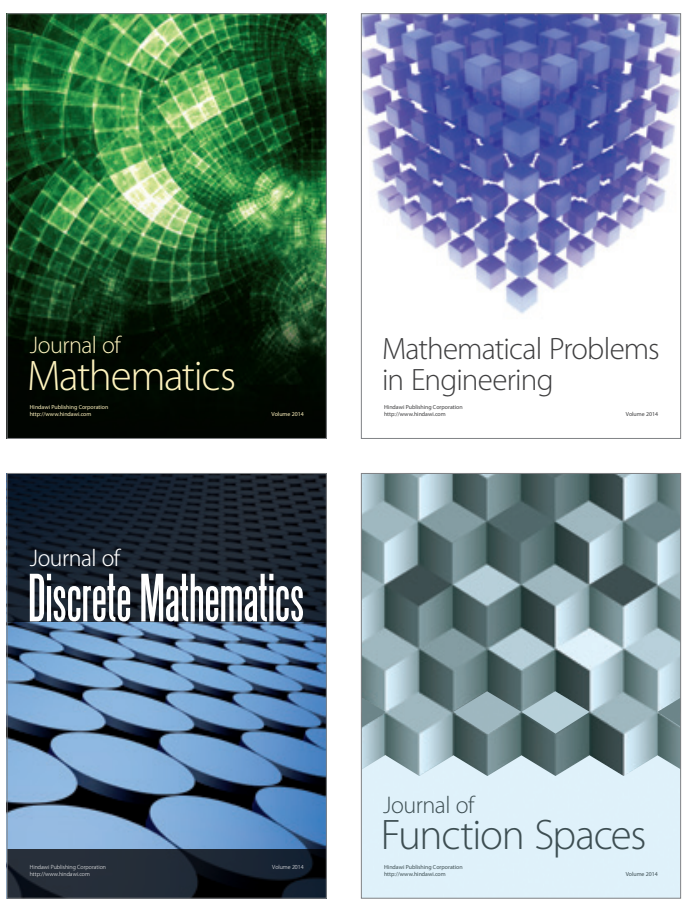

Mathematical Problems in Engineering
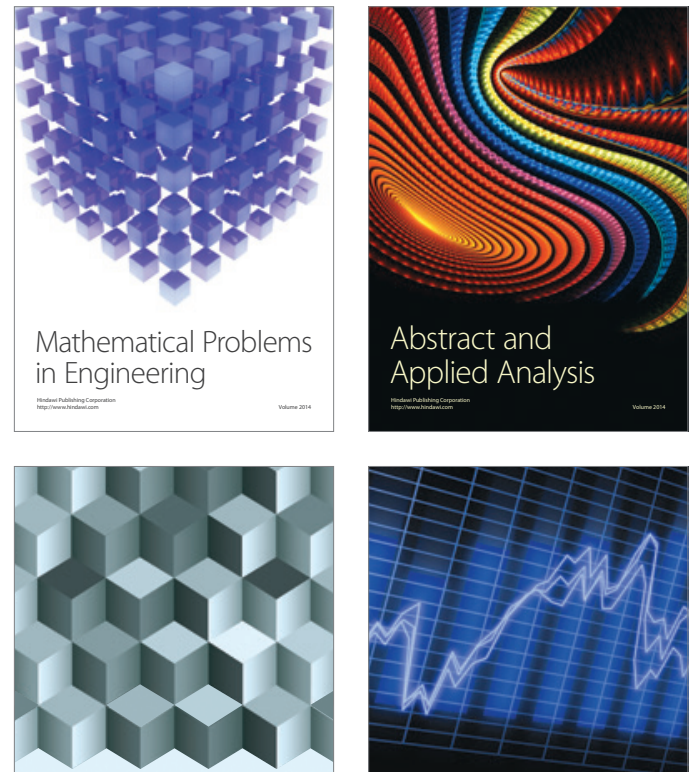

Journal of

Function Spaces

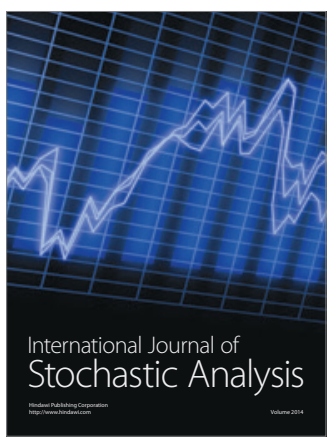

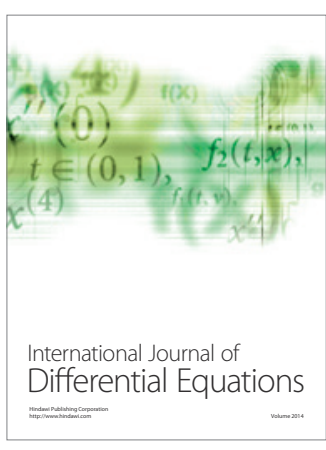
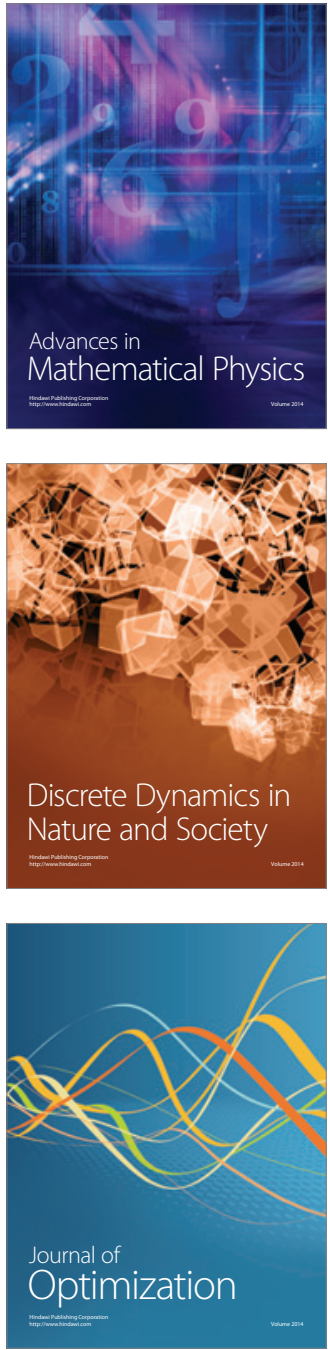